\title{
LIGHT SCATTERING INVESTIGATIONS OF MAGNETIC ANISOTROPIES IN ULTRATHIN EPITAXIAL Co FILMS
}

\author{
B. Hillebrands, P. Krams, J. Fassbender, C. Mathieu, G. Güntherodt
}

2. Physikalisches Institut, RWTH Aachen, 52056 Aachen, Germany

\section{R. JUNGBLUT AND M.T. JOHNSON}

Philips Research, P.O. Box 80000, 5600JA Eindhoven, The Netherlands

\begin{abstract}
Using Brillouin light scattering, the properties of magnetic anisotropies in ultrathin epitaxial Co films grown on single-crystal $\mathrm{Cu}$ substrates are investigated. All relevant magnetic anisotropy contributions are determined, in silu in an ultrahigh vacuum system for the (001)-oriented films, as well as $e x$ situ for other film orientations, which are covered with a $\mathrm{Cu}$ cap layer. For (001)-oriented films we find a large in-plane anisotropy contribution of fourfold symmetry. For this contribution the volume and the surface part cancel to zero at the same thickness as found for the onset of ferromagnetic order. This finding is indicative for a stabilization of ferromagnetic order in $\mathrm{Co} / \mathrm{Cu}(001)$ by in-plane anisotropy contributions. In ( $\left.\begin{array}{lll}1 & 1 & 13\end{array}\right)$-oriented films, which are composed of (001)-terraces aligned along the [110]-direction and spaced by 6.5 atomic distances, an additional uniaxial in-plane anisotropy contribution is found which is identified as being of magneto-elastic origin. For (110)-oriented films three ranges of thickness dependence of the in-plane and out-of-plane anisotropy contributions are found which correlate in the thin-film regime with the growth properties. (111)-oriented films show a linear dependence of the perpendicular anisotropy on the reciprocal film thickness over the full investigated thickness range of 2 to $20 \AA$.
\end{abstract}

PACS numbers: 75.30.Gw, 75.30.Pd, 75.30.Ds, 78.35.+c

\section{Introduction}

Magnetic properties of ultrathin films of $3 d$ transition metals are inherently determined by magnetic anisotropies which are found to be larger by up to three orders of magnitude in energy per atom compared to the corresponding bulk materials. However, although the experimental database on anisotropy constants is steadily increasing, little is known up to now about the origin of the observed large anisotropy values and their interplay with other magnetic properties like 
the Curie temperature or the critical minimum thickness for ferromagnetic order. Among the currently discussed anisotropy mechanisms, apart from the shape anisotropy, contributions from Néel-type surface anisotropies [1], contributions form magneto-elastic interactions due to elastic strains [2] and magnetocrystalline anisotropy contributions are considered.

In this paper we investigate all magnetic anisotropy contributions found in $\mathrm{Co}$ layers of various orientations prepared on single-crystal $\mathrm{Cu}$ substrates. We will show that in-plane anisotropy contributions are closely connected to the mechanism for stabilizing ferromagnetic order at room temperature for Co films of (100)-orientation. Specific information is obtained from Co (1 1113$)$-oriented films which can be regarded as (001)-oriented films tilted by an angle of $6.2^{\circ}$ about the in-plane [110] axis: due to the induced twofold in-plane symmetry, an additional in-plane uniaxial anisotropy contribution is found and identified as being of magneto-elastic origin caused by the elastic strain fields generated by the lattice mismatch at the substrate-film interface. Finally, we discuss the presence of bulk magnetocrystalline anisotropies and its disappearance for thin films which can be studied in (110)-oriented films.

The experimental method of choice is Brillouin light scattering from thermally activated spin waves. Due to the precession of the magnetic moments, forming the spin wave, the torques acting on the magnetization, i.e. the two anisotropy field components perpendicular to the mean direction of magnetization, are sensed. The spin wave frequencies are further sensitive to the magnitudes and directions of the magnetization and the spin-wave wave vector as well as to the film thickness. Compared to static and macroscopic methods Brillouin light scattering has the specific advantage of measurements with high local resolution (laser spot is $\approx 100 \mu \mathrm{m}$ in diameter) as well as monolayer sensitivity.

\section{Anisotropies in ultrathin magnetic layers}

Magnetic anisotropies reflect to a large degree the symmetry of the system as well as acting symmetry breaking mechanisms. We distinguish between the shape anisotropy, the magnetocrystalline anisotropy due to a coupling of the magnetization to the crystallographic symmetry (symmetry of electronic band structure), surface anisotropies due to the lack of translational symmetry along the film normal, as well as magneto-elastic anisotropies due to elastic strain fields, which break the crystallographic symmetry and result in a lower symmetry class of the films. The coordinate system is chosen such that the $\hat{z}$-axis is aligned along the film normal and the $\hat{x}$-axis is parallel to the lowest-indexed in-plane direction. In the following we write the free magnetic energy density, $F$, in terms of direction cosines $\alpha_{i}$ of the saturation magnetization, $\boldsymbol{M}_{\mathrm{s}}$, which are the components of the unit vector $\boldsymbol{\alpha}$ pointing into the direction of $\boldsymbol{M}_{\mathbf{s}}$. We denote volume anisotropies by upper case letters and surface anisotropies by lower case letters. If appropriate, the rotational symmetry of an anisotropy constant about a symmetry axis is denoted by a superscript in brackets.

The magnetocrystalline anisotropy is caused by the coupling of the free energy, $F_{\text {ani }}$, of the magnetization as a function of the direction of $M$ to the crystallographic symmetry due to spin-orbit coupling. In cubic symmetry $F_{\text {ani }}$ is given by

$$
F_{\text {ani }}=K_{1}\left(\alpha_{x^{\prime}}^{2} \alpha_{y^{\prime}}^{2}+\alpha_{y^{\prime}}^{2} \alpha_{z^{\prime}}^{2}+\alpha_{z^{\prime}}^{2} \alpha_{x^{\prime}}^{2}\right)
$$


with $K_{1}$ the anisotropy constant, which is of fourth order, and with $\alpha_{i}$, the direction cosines of the magnetization, here defined with respect to the crystallographic $\langle 100\rangle$ principal axes. Surface anisotropies arise due to the broken translational symmetry along the film normal. Due to the pair bonding model of Néel $[1,3]$ they can be calculated by performing the dipolar sums of the magnetic moments located at the interfaces, although the obtained values do not well agree, even in sign, with experimental results. In lowest orders the free surface energy density, $\sigma_{\text {surf }}$ is given by

$$
\sigma_{\text {surf }}=-k_{\mathrm{s}} \alpha_{z}^{2}+k_{\mathrm{p}}^{(2)} \alpha_{x}^{2}+k_{\mathrm{p}}^{(4)} \alpha_{x}^{2} \alpha_{y}^{2}
$$

with $k_{\mathrm{s}}$ the out-of-plane anisotropy constant. The constants $k_{\mathrm{p}}^{(2)}$ and $k_{\mathrm{p}}^{(4)}$ describe the first two non-vanishing orders of the in-plane surface anisotropy. They are of second and fourth order in $\alpha_{i}$ and they correspond to two- and fourfold symmetry about the film normal, respectively. The signs are chosen in the usual way, i.e., a positive sign of $k_{\mathrm{s}}$ indicates a corresponding easy axis perpendicular to the film and a positive sign of $k_{\mathrm{p}}^{(2)}$ and $k_{\mathrm{p}}^{(4)}$ indicates that the [100] direction is hard. Since the second term in Eq. (2) is of second order in $\alpha_{i}$, it only exists for surfaces of not higher than twofold symmetry.

If the film thickness is smaller than the static exchange correlation length $(\approx 50 \AA$ for $\mathrm{Co}$ ) the magnetization is homogeneous across the film. Then the surface torques caused by surface anisotropies acting on the moments located at the surfaces can be converted to volume torques acting on the entire film magnetization. This corresponds to a replacement of the surface energy density, $\sigma_{\text {surf }}$, with the volume energy density

$$
F_{\text {surf }}=\frac{2}{d} \sigma_{\text {surf }}
$$

with the factor of two counting the two surfaces of the film. Rado [4] and Gradmann et al. [5] give estimates for the range of validity for Eq. (3).

Magneto-elastic anisotropies arise from in-plane strains caused by the lattice mismatch at the substrate-film interface. We consider the case that the elastic strains $\varepsilon_{i}$ are not released due to, e.g., formation of misfit dislocations.

With $\varepsilon_{i}$ the strain components in the film, $b_{i j}$ the magneto-elastic tensor and $\alpha_{i}$ the magnetization direction cosines for axes $\hat{x}_{i}$, the free magneto-elastic energy density is given by

$$
F_{\mathrm{me}}=\sum_{i j k l} b_{i j k l} \alpha_{i} \alpha_{j} \varepsilon_{k l} .
$$

The tensor constants $b_{i j k l}$ as well as those of the elastic constants, $c_{i j k l}$, needed to calculate $b_{i j k l}$ from magnetostriction constants, are rotated from the crystallographic reference frame into the oriented layer frame. At the substrate-film interface the strains must accommodate the in-plane mismatch

$$
a_{\mathrm{s}}=a_{\mathrm{f}}\left(1+\varepsilon_{i i}\right), \quad i=1,2
$$

with $a_{\mathrm{s}}\left(a_{\mathrm{f}}\right)$ the lattice parameter of the substrate (film). The out-of-plane strain $\varepsilon_{33}$ is obtained by minimizing the free elastic energy in the film

$$
F_{\text {elast }}=\sum_{i j k l} c_{i j k l} \varepsilon_{i j} \varepsilon_{k l}
$$


All other strain components are zero. The strain-induced anisotropy contributions are obtained from Eq. (4) by a comparison to a general anisotropy energy expression of the form

$$
F_{\text {ani }}=-K_{\mathrm{s}} \alpha_{3}^{2}+K_{\mathrm{p}} \alpha_{1}^{2}
$$

with $K_{\mathrm{p}}\left(K_{\mathrm{s}}\right)$ the strain-induced uniaxial in-plane (out-of-plane) anisotropy constant. The signs in Eq. (7) are chosen following the usual convention that a positive sign denotes a hard (easy) axis for the in-plane (out-of-plane) anisotropy constant. Equation (7) is formally close to Eq. (2), which describes surface anisotropies, since in both cases the lowest non-vanishing orders are of second order. The results for different types of film orientations are tabulated in Refs. [6, 7].

For ultrathin Co films of two- or fourfold symmetry about the surface normal, the free energy contribution, $F_{\text {ani }}$, due to anisotropies is expressed in the lowest order as

$$
\begin{aligned}
F_{\text {ani }} & =\left(K_{\mathrm{p}}^{(2)}+\frac{2}{d} k_{\mathrm{p}}^{(2)}\right) \sin ^{2} \theta \cos ^{2}\left(\phi-\phi_{0}\right)+\frac{1}{4}\left(K_{\mathrm{p}}^{(4)}+\frac{2}{d} k_{\mathrm{p}}^{(4)}\right) \sin ^{2} 2 \phi \sin ^{4} \theta \\
& -\left(K_{\mathrm{s}}+\frac{2}{d} k_{\mathrm{s}}\right) \cos ^{2} \theta
\end{aligned}
$$

$\theta$ and $\phi$ are the polar and azimuthal angle of the direction of magnetization, $\boldsymbol{M}$. Here $\phi$ is measured against the in-plane [100]-axis. The first term on the right hand side is the in-plane anisotropy of twofold symmetry about the film normal with the angle $\phi_{0}$ measuring a possible incline of the uniaxial reference axis to the [100]-axis. The second term is the corresponding term due to a fourfold symmetry and the last term is the out-of-plane anisotropy. All terms are further splitted between volume and surface contributions.

\section{Spin waves in ultrathin magnetic layers}

In thin ferromagnetic films, at room temperature, there exists a thermally activated, so-called Damon-Eshbach spin wave excitation. Its energy is primarily determined by the dipolar spin-spin interaction, since the wavelength of the order of $3000 \AA$ is large compared to interatomic distances [8]. If the product of the film thickness $d$ and the wave vector $q_{\|}$is small compared to unity, the frequency $\omega$ of the mode can analytically be expressed as [9]:

$$
\begin{aligned}
& \left(\frac{\omega}{\gamma}\right)^{2}=\left[H+4 \pi M_{\mathrm{s}}\left(1-\frac{1}{2} q_{\|} d\right)+\frac{\partial^{2} F_{\mathrm{ani}}}{M_{\mathrm{s}} \partial \theta^{2}}\right] \\
& \quad \times\left[H+\frac{\partial^{2} F_{\text {ani }}}{M_{\mathrm{s}} \partial \phi^{2}}+2 \pi M_{\mathrm{s}} q_{\|} d \sin ^{2}\left(\phi-\phi_{q}\right)\right] .
\end{aligned}
$$

Here $F_{\text {ani }}$ is the magnetic free anisotropy energy, and $\phi-\phi_{q}$ is the angle between the direction of magnetization and the spin-wave wave vector.

From Eq. (9) it follows that the Damon-Eshbach mode is sensitive to the product $q_{\|} d$, the strength and direction of the applied field, the saturation magnetization, and, in particular, to anisotropy contributions. Die to the latter an investigation of the Damon-Eshbach mode is very suitable to yield information about the anisotropy contributions present in magnetic films. 


\section{Sample preparation and characterization}

All (001)- and (1 113$)$-oriented samples were prepared in an ultrahigh vacuum chamber of base pressure better than $5 \times 10^{-11}$ mbar by $\mathrm{e}^{-}$-beam evaporation at a pressure of $2 \times 10^{-10}$ mbar. The details are reported elsewhere [10,11]. Special emphasis was placed on the preparation of smooth films with minimum interdiffusion at the $\mathrm{Co} / \mathrm{Cu}$ interface. In the case of the (001)-orientation, films of $3 \mathrm{ML}$ (monolayers) thickness were prepared at different deposition temperatures, $T_{\text {dep }}$, between $20^{\circ} \mathrm{C}$ and $140^{\circ} \mathrm{C}$, yielding the best results at $T_{\text {dep }}=85^{\circ} \mathrm{C}$. For the latter deposition temperature the Auger-intensity ratio of the signals from the Co $656 \mathrm{eV}$ and $716 \mathrm{eV}$ lines to those of the $\mathrm{Cu} 849 \mathrm{eV}$ and $920 \mathrm{eV}$ lines has a maximum indicating a uniform film with minimum interdiffusion and maximum interface smoothness. From the spin wave spectra it is found that the "magnetic quality" of the films is also best at $T_{\text {dep }}=85^{\circ} \mathrm{C}$. For this deposition temperature the magnetization is found to rotate as a function of field strength in the most uniform way from the easy [110]-direction into the hard [100]-direction along which the external field is applied (see below). For the $\left(\begin{array}{lll}1 & 1 & 13\end{array}\right)$ film orientation the optimum deposition temperature is $300 \mathrm{~K}$. The (110)- and (111)-oriented films were grown in the form of wedge-shaped samples at room temperature at the Philips Research Laboratories in a manner similar to that described in Ref. [12].

\section{Experimental}

The Brillouin light scattering measurements of the spin wave excitations were performed in situ for (001)-oriented films at room temperature focusing the $\lambda=5145 \AA$ laser light of an $\mathrm{Ar}^{+}$ion laser of up to $120 \mathrm{~mW}$ intensity through a viewport onto the sample and collecting through the same viewport the inelastically scattered light in backscattering geometry. The maximum strength of the external field applied parallel to the film plane and perpendicular to the spin-wave wave vector was $1.8 \mathrm{kOe}$. The measurements for other orientations were performed in the same geometry ex situ in an applied field of up to $20 \mathrm{kOe}$. The frequency analysis was performed using a computer-controlled automated high-contrast $(3+3)$-pass tandem Fabry-Perot interferometer [13]. The measurement time per spectrum was typically $30-120 \mathrm{~min}$. The wave vector $q$ of spin waves tested in the experiment was $1.73 \times 10^{5} \mathrm{~cm}^{-1}$. In addition, the magnetic hysteresis loop was measured in situ with the transverse magneto-optic Kerr effect using a $\mathrm{He}-\mathrm{Ne}$ laser.

\section{6. $\mathrm{Co} / \mathrm{Cu}(111)$ : perpendicular anisotropy}

We first discuss results on Co (111)-oriented films, which exhibit large anisotropies with the film normal as the easy axis. Figure 1 shows the measured spin wave frequency, $\nu$, as a function of the film thickness measured on a single wedge-shaped sample. An extrapolation of the data to $\nu=0$ yields a critical thickness, $d_{\mathrm{c}}=3 \AA$, below which the assumed magnetization orientation (parallel to the film plane) is not stable at the given applied field strength. From the experimental data the bulk hexagonal anisotropy constant, $K_{1}^{\text {hex }}$, and the out-of-plane surface anisotropy constant, $k_{\mathrm{s}}^{(2)}$, are obtained by a least squares fit of Eq. (9) 


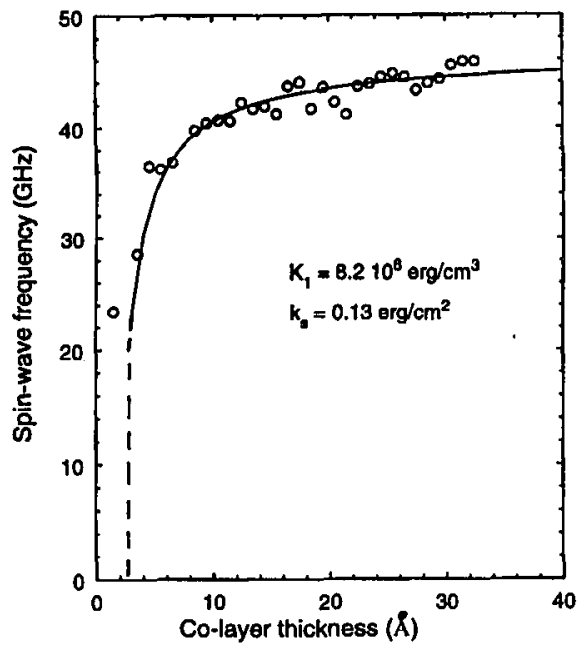

Fig. 1. Measured (circles) and fitted (full line) spin wave frequencies for Co(111) films on $\mathrm{Cu}(111)$ in an external field of $12 \mathrm{kOe}$ applied parallel to the film plane.

TABLE

Obtained anisotropy constants for the (001)-, (1 11 13)- and (111)-orientations. A cover layer is noted in brackets. The parameters are defined in the body of the text. $K_{1}^{\text {thex }}$ is the usually defined hexagonal Co bulk anisotropy constant used for the (111)-orientation.

\begin{tabular}{|c|c|c|c|c|c|c|}
\hline & $\begin{array}{c}K_{\mathrm{p}}^{(2)} \\
{\left[10^{6}\right.} \\
\left.\mathrm{erg} / \mathrm{cm}^{3}\right]\end{array}$ & $\begin{array}{c}k_{\mathrm{p}}^{(2)} \\
{\left[10^{-3}\right.} \\
\left.\mathrm{erg} / \mathrm{cm}^{2}\right]\end{array}$ & $\begin{array}{l}K_{\mathrm{p}}^{(4)} \\
{\left[10^{6}\right.} \\
\left.\mathrm{erg} / \mathrm{cm}^{3}\right]\end{array}$ & $\begin{array}{c}k_{\mathrm{p}}^{(4)} \\
{\left[10^{-3}\right.} \\
\left.\mathrm{erg} / \mathrm{cm}^{2}\right]\end{array}$ & $\begin{array}{l}K_{\mathrm{s}}, K_{1}^{\text {hex }} \\
{\left[10^{6}\right.} \\
\left.\mathrm{erg} / \mathrm{cm}^{3}\right]\end{array}$ & $\begin{array}{c}k_{\mathrm{s}} \\
{\left[\mathrm{erg} / \mathrm{cm}^{2}\right]}\end{array}$ \\
\hline $\begin{array}{l}\text { Co(001) } \\
\text { (no cap layer) }\end{array}$ & - & - & $-2.3 \pm 0.15$ & $34 \pm 4$ & $\approx 0$ & $-0.46 \pm 0.09$ \\
\hline $\begin{array}{l}\mathrm{Co}(001) \\
(2 \mathrm{ML} \mathrm{Cu})\end{array}$ & - & - & $-2.2 \pm 0.15$ & $31 \pm 3$ & $\approx 0$ & $0.15 \pm 0.04$ \\
\hline $\begin{array}{l}\text { Co(001)/vac. } \\
\text { interface }\end{array}$ & & & & $32 \pm 3$ & & $-1.06 \pm 0.17$ \\
\hline $\begin{array}{l}\mathrm{Co}(001) / \mathrm{Cu} \\
\text { interface }\end{array}$ & & & & $32 \pm 3$ & & $0.15 \pm 0.04$ \\
\hline $\begin{array}{l}\mathrm{Co}\left(\begin{array}{lll}1 & 1 & 13\end{array}\right) \\
(20 \mathrm{ML} \\
(2 \mathrm{Cu})\end{array}$ & $0.60 \pm 0.07$ & $-9 \pm 2$ & $-0.65 \pm 0.02$ & $12 \pm 2$ & $-5.0 \pm 0.6$ & $\approx 0$ \\
\hline $\begin{array}{l}\mathrm{Co}(111) \\
(\mathrm{BLS})^{*} \\
(20 \mathrm{ML} \mathrm{Cu})\end{array}$ & & & & & $8.2 \pm 0.8$ & $0.13 \pm 0.04$ \\
\hline $\begin{array}{l}\mathrm{Co}(111) \\
(\mathrm{MOKE})^{* *} \\
(20 \mathrm{ML} \mathrm{Cu})\end{array}$ & & & & & $8.5 \pm 0.6$ & $0.21 \pm 0.04$ \\
\hline
\end{tabular}

${ }^{*}$ Brillouin light scattering. ${ }^{* *}$ Magneto-optical Kerr effect. 
to the experimental frequency values. We obtain $K_{1}^{(\text {hex })}=8.2 \times 10^{6} \mathrm{erg} / \mathrm{cm}^{3}$ and $k_{\mathrm{s}}=0.13 \mathrm{erg} / \mathrm{cm}^{2}$ [14]. Independent measurements of the anisotropy of the wedge-shaped sample were performed using the magneto-optic Kerr effect [14]. The Brillouin light scattering results and the magneto-optic Kerr measurements provide values for $K_{1}$ and $k_{s}$ in close agreement (see Table). Our results indicate a somewhat larger interface anisotropy, but a considerable reduced volume anisotropy contribution compared to recent results of Kohlhepp et al. [15]. The spin wave frequencies are found to be isotropic as a function of the in-plane direction of the applied field, indicating that there are no in-plane anisotropy contributions.

\section{7. $\mathrm{Co} / \mathrm{Cu}(001)$ : stabilization of ferromagnetic order by in-plane anisotropies}

We now address a problem of great interest. Will magnetic anisotropies stabilize ferromagnetic order in ultrathin films? According to the theorem of Mermin and Wagner [16] a ferromagnetic ground state cannot exist at finite temperatures in an isotropic two-dimensional Heisenberg system with short-range interactions. Several mechanisms have been proposed to account for the experimentally observed existence of ferromagnetic order in two-dimensional systems [17-21]. Among the mechanisms the stabilization of ferromagnetic order due to dipolar interactions or due to magnetic anisotropies are currently discussed most.

In the following we discuss the determination of all relevant magnetic volume and surface anisotropy contributions at room temperature in ultrathin epitaxial (001)-oriented films of fcc $\mathrm{Co}$ on $\mathrm{Cu}(001)$ substrates by means of Brillouin light scattering $[10,22]$. We show evidence that in this system magnetic in-plane anisotropy contributions stabilize ferromagnetic order at room temperature.

In the in situ light scattering experiments the external magnetic field of up to $1.8 \mathrm{kOe}$ is applied in the film plane along the magnetic hard [100] axis.

Ferromagnetic order is observed for film thickness $d$ larger than $d_{\mathrm{c}}=$ $1.6 \pm 0.3 \mathrm{ML}$ for uncovered Co films and $d_{\mathrm{c}}=1.9 \pm 0.3 \mathrm{ML}$ for Cu covered Co films $[10,22]$. We identify the onset of ferromagnetic order by the existence of a remanent magnetization resulting in a coercive field, which is tested by magneto-optical Kerr effect, as well as by the existence of spin waves at zero applied fields [23]. The obtained coercive field is $(81 \pm 14)$ Oe for the uncovered film and $(66 \pm 11) \mathrm{Oe}$ for the covered film.

In situ magneto-optical Kerr effect measurements of the remanent field as a function of the in-plane direction of the external field clearly show that the acting in-plane anisotropies are of fourfold symmetry about the film normal in the lowest order, i.e. the first right hand side term in Eq. (8) and thus $K_{\mathrm{p}}^{(2)}$ and $k_{\mathrm{p}}^{(2)}$ are zero.

By applying the external magnetic field along the magnetic hard [100]-axis, one can probe the magnetic anisotropies by studying the rotation of the magnetization with increasing field into the direction of the applied field via the corresponding change in the spin wave frequency. Upon increasing the applied field the spin wave frequency first decreases due to the change in $\phi$, until a critical field strength, $H_{\text {crit }}$, is reached. For $H>H_{\text {crit }}$, the magnetization and the applied field are collinear and the spin wave frequency increases nearly linear with further increasing field. $H_{\text {crit }}$ is a measure of the in-plane anisotropy. 
For the films considered here the product of the film thickness $d$ and the wave vector $q_{\|}$is small compared to unity. Therefore we may use Eq. (9) for calculating the spin wave frequency of the Damon-Eshbach mode. From a least squares fit of Eq. (9) to the measured spin wave frequencies as a function of the applied field and the Co film thickness the saturation magnetization and the anisotropy constants $K_{\mathrm{p}}^{(4)}, k_{\mathrm{p}}^{(4)}$ and $k_{\mathrm{s}}$ are determined. For all studied films with $d>d_{\mathrm{c}}$ the saturation magnetization does not deviate from the bulk value of $17.9 \mathrm{kG}$ within the limit of accuracy of $5 \%$. The result is corroborated by finding a linear increase in the Kerr rotation angle with increasing film thickness measured in situ on the same films as used for the light scattering studies.

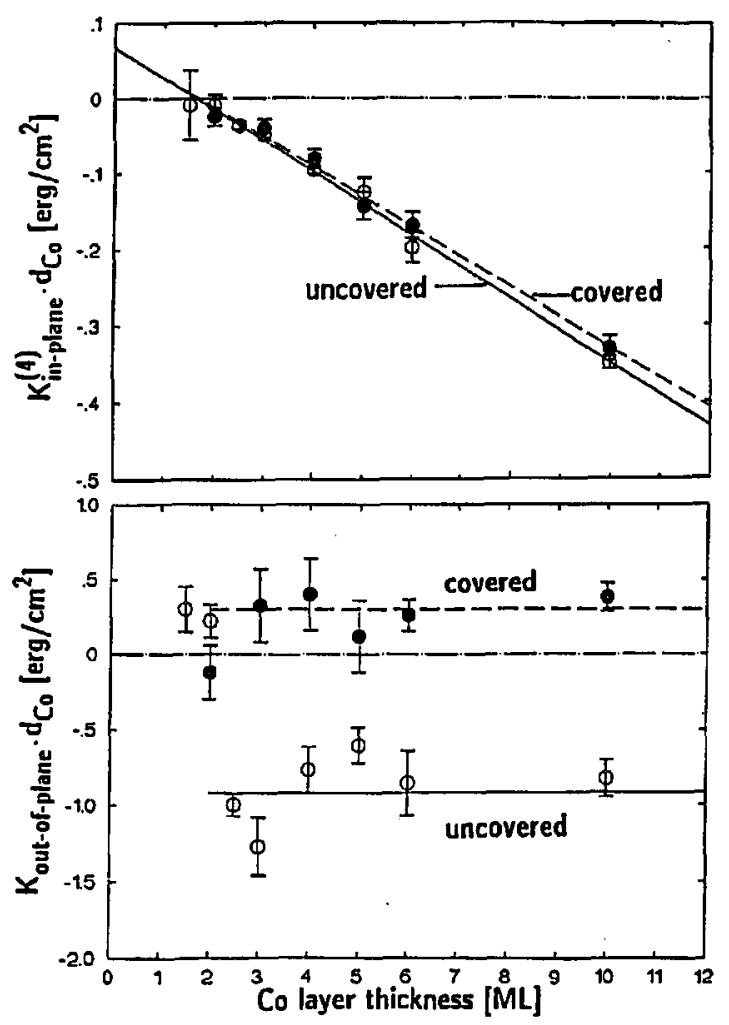

Fig. 2. Measured values of the in-plane anisotropy of fourfold symmetry, $K_{\text {in-plane }}^{(4)}$ (upper part), and the out-of-plane anisotropy, $K_{\text {out-of-plane (lower part), multiplied }}$ with the film thickness, $d$, as a function of $d$ in $\mathrm{ML}$ for $\mathrm{Co} / \mathrm{Cu}(001)$ without (open circles) and with (full circles) a $2 \mathrm{ML} \mathrm{Cu}$ cover layer. A fit to the theory is shown for data of $\mathrm{Co} / \mathrm{Cu}(001)$ (full line) and for data of $\mathrm{Cu} / \mathrm{Co} / \mathrm{Cu}(001)$ (dashed line).

Figure 2 shows the obtained anisotropy values as a function of film thickness. for uncovered Co films (open symbols) and Co films covered with $2 \mathrm{ML} \mathrm{Cu}$ (closed symbols). In the upper part the total in-plane anisotropy multiplied with the film 
thickness $d$,

$$
K_{\text {in-plane }}^{(4)} d=K_{\mathrm{p}}^{(4)} d+2 k_{\mathrm{p}}^{(4)}
$$

is shown. The lower part displays the out-of-plane anisotropy constant, $K_{\text {out-of-plane: }}$ :

$$
K_{\text {out-of-plane }} \mathrm{d}=K_{\mathrm{s}}^{(2)} \mathrm{d}+2 k_{\mathrm{s}}^{(2)} .
$$

In these graphs the slopes are determined by the volume anisotropy constants, and the intercepts with the $y$-axis are equal to twice the corresponding surface anisotropy constants. From the fit to the experimental data we find $K_{\mathrm{s}}^{(2)}=0$ both for the uncovered and covered films. The average value of $k_{\mathbf{s}}=(-0.46 \pm$ $0.09) \mathrm{erg} / \mathrm{cm}^{2}$ for uncovered Co films changes to $k_{\mathrm{s}}=(0.15 \pm 0.04) \mathrm{erg} / \mathrm{cm}^{2}$ upon covering the Co films by $2 \mathrm{ML} \mathrm{Cu}$. The negative sign indicates that the surface normal is a magnetic hard axis for this anisotropy contribution.

Of particular interest are the properties of $K_{\text {in-plane. From the slope and }}$ the intercept of the straight lines in Fig. 2 we yield for the volume $\left(K_{\mathrm{p}}^{(4)}\right)$ and the surface $\left(k_{\mathrm{p}}^{(4)}\right)$ contribution values of $K_{\mathrm{p}}^{(4)}=(-2.3 \pm 0.15) \times 10^{6} \mathrm{erg} / \mathrm{cm}^{3}$ and $k_{\mathrm{p}}^{(4)}=(0.034 \pm 0.004) \mathrm{erg} / \mathrm{cm}^{2}$ for the uncovered films, and $K_{\mathrm{p}}^{(4)}=(-2.2 \pm$ $0.15) \times 10^{6} \mathrm{erg} / \mathrm{cm}^{3}$ and $k_{\mathrm{p}}^{(4)}=(0.031 \pm 0.003) \mathrm{erg} / \mathrm{cm}^{2}$ for the Co layers covered with $2 \mathrm{ML} \mathrm{Cu}$. Due to their opposite sign, the contributions of $K_{\mathrm{p}}^{(4)}$ and $k_{\mathrm{p}}^{(4)}$ to

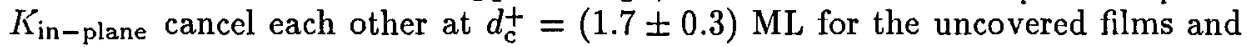
at $d_{c}^{+}=(1.6 \pm 0.3) \mathrm{ML}$ for the Cu covered films. We would like to point out that $d_{c}^{+}=d_{c}$ within the experimental error for both covered and uncovered Co films, although both quantities are determined by independent experiments. The obtained anisotropy values are summarized in Table.

Assuming that the Co films covered by $2 \mathrm{ML} \mathrm{Cu}$ have symmetric surfaces with the same anisotropy constants, the Co surface anisotropy constants are independently accessible for the vacuum and the $\mathrm{Cu}$ side by comparing the data of the uncovered to the covered Co films. Since $K_{\text {in-plane }}$ does not significantly change upon covering the $\mathrm{Co}$ layer by $\mathrm{Cu}$, both the $\mathrm{Co} / \mathrm{Cu}$ and $\mathrm{Co} /$ vacuum surface have the same value of $k_{\mathrm{p}}^{(4)}=(0.032 \pm 0.003) \mathrm{erg} / \mathrm{cm}^{2}$. The out-of-plane anisotropy constant, $k_{\mathrm{s}}$, was found to be $k_{\mathrm{s}}=(-1.06 \pm 0.17) \mathrm{erg} / \mathrm{cm}^{2}$ for the Co/vacuum interface and $k_{\mathrm{s}}=(0.15 \pm 0.04) \mathrm{erg} / \mathrm{cm}^{2}$ for the Co/Cu interface (see also Table).

From the observed agreement between the critical thickness for ferromagnetic order, $d_{c}$, with the thickness $d_{c}^{+}$, at which the contributions to the in-plane anisotropy cancel, it is concluded that the symmetry breaking interaction for stabilizing ferromagnetic order in $\mathrm{Co}(001)$ films at room temperature is indeed given by the magnetic in-plane anisotropy contribution [10,22]. It should be pointed out that this argument is backed by the fact that $d_{c}^{+}$may also be obtained from an extrapolation of $K_{\text {in-plane }}(d)$ from data with film thicknesses significantly larger than $d_{c}$, thus ruling out structural and/or magnetic percolation effects near $d_{c}^{+}$. The out-of-plane anisotropy, described by the thickness independent parameter $k_{s}$ does not support the stabilization. The data clearly indicate that in contrast to the critical thickness, $d_{\mathrm{c}}$, and to the in-plane anisotropy constant $K_{\mathrm{in} \text {-plane }}, k_{\mathrm{s}}$ is very sensitive to the presence of a $\mathrm{Cu}$ overlayer.

The origin of $k_{s}$ and $K_{\text {in-plane, }}$ in particular their different sensitivity to the presence of a $\mathrm{Cu}$ overlayer, is presently not well understood. The thickness-inde-

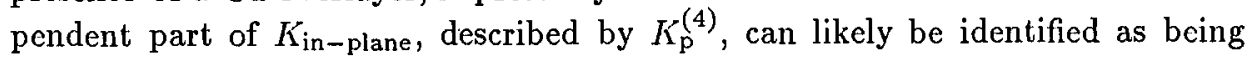


magnetocrystalline in origin due to its sign and magnitude (see below). However, in order to compare $K_{\mathrm{p}}^{(4)}$ to a crystalline anisotropy of cubic symmetry appropriate for (100)-oriented Co films of larger thickness, a thickness-independent uniaxial perpendicular anisotropy contribution of appropriate size must be considered in Eq. (8) in order to make the volume anisotropy contribution invariant against all cubic symmetry transformations. No evidence has been found for such a contribution from the light scattering data for the investigated film thickness regime of $1 \mathrm{ML} \leq d \leq 10 \mathrm{ML}$.

It might be speculated that the values of $k_{s}$ and $K_{\text {in-plane }}$ are due to the elastic strain fields parallel to the film induced by the lattice mismatch of $1.9 \%$ at the $\mathrm{Co} / \mathrm{Cu}$ interface. Hence the values of $k_{\mathrm{s}}$ and $K_{\text {in-plane }}$ would be proportional to the interface strain. An estimate for the out-of-plane magneto-elastic anisotropy yields values of the same order of magnitude, in particular the same sign as measured for $k_{\mathrm{s}}$. For this estimate the fcc Co magnetostriction constants extrapolated for Co rich fcc Pd-Co alloys were used [24]. However, since the Co strains are not expected to largely change upon coverage with a $\mathrm{Cu}$ overlayer the finding of a very sensitive dependence of $k_{\mathrm{s}}$ on a $\mathrm{Cu}$ overlayer is in contradiction to the assumption of magneto-elastic contributions to $k_{\mathrm{s}}$. In order to estimate a magneto-elastic contribution to $K_{\text {in }}$-plane , higher order magnetostriction constants would have to be included which are not available and which would need to be very large, which is unlikely. On the other hand, $k_{\mathrm{s}}$ may be related to the spacing between the surface Co layer and the underlying atomic Co layer which is known to be very sensitive to the $\mathrm{Cu}$ overlayer. Detailed total energy calculations are highly desirable to clarify the origin of these anisotropies in this two-dimensional model system.

\section{8. $\mathrm{Co} / \mathrm{Cu}\left(\begin{array}{lll}1 & 1 & 13\end{array}\right)$ : induced uniaxial anisotropy}

Additional information regarding the physical origin of thin film anisotropies is obtained by studying Co films on (1113)-oriented Cu substrates [22]. In this system the (001)-surface is tilted by an angle of $6.2^{\circ}$ about the in-plane [110] axis. The (1 11 13)-surface consists of (001)-oriented terraces with a verage width of 6.5 atomic distances, separated by monoatomic steps aligned with the [110]-axis. Here, due to the induced twofold in-plane symmetry, an additional in-plane uniaxial anisotropy contribution, described by the constant $K_{\mathrm{p}}^{(2)}$ is found. The easy axis was found to lie along the steps $\left(\phi_{0}=45^{\circ}\right.$ in Eq. (8)) [25].

By use of Eq. (8) the measured spin wave frequencies were fitted using Eq. (9) with the anisotropy constants as fit parameters. In the least squares fit much care has been taken to control the errors and correlations between the parameters. Performing the same type of analysis as in the case of $\mathrm{Co}(100)$ films, critical thicknesses are deduced from the data, at which the corresponding volume and surface anisotropies cancel to zero. For the twofold anisotropy this thickness is $d_{\mathrm{c}}^{(2)}=(2.9 \pm 0.5) \mathrm{ML}$ for the uniaxial anisotropy and $d_{\mathrm{c}}^{(4)} \mathrm{ML}$ for the fourfold anisotropy. We find that within the experimental error $d_{\mathrm{c}}^{(2)}=d_{\mathrm{c}}^{(4)}=d_{\mathrm{c}}$ with $d_{\mathrm{c}}$ the independently determined minimum thickness of $2 \mathrm{ML}$ for the onset of ferromagnetic order [26]. We would like to point out that although the film growth mode is very different for the (1113) surface orientation compared to the (001)-orientation $[10,11,27]$ the critical thickness for the onset of ferromagnetic order is the same within the error margins for both orientations. 
From the data analysis we find for the twofold in-plane anisotropy $K_{\mathrm{p}}^{(2)}=$ $(6.0 \pm 0.7) \times 10^{5} \mathrm{erg} / \mathrm{cm}^{3}$ and $k_{\mathrm{p}}^{(2)}=(-0.009 \pm 0.002) \mathrm{erg} / \mathrm{cm}^{(2)}$, and for the fourfold contribution $K_{\mathrm{p}}^{(4)}=(-6.5 \pm 0.2) \times 10^{(5)} \mathrm{erg} / \mathrm{cm}^{3}$ and $k_{\mathrm{p}}^{(4)}=(0.012 \pm$ $0.002) \mathrm{erg} / \mathrm{cm}^{2}$.

For $d>d_{\mathrm{c}}^{(2)}$ the [110] direction (parallel to the steps) is the easy axis for $K_{\mathrm{in}-\text { plane }}^{(2)}$, and for $d>d_{\mathrm{c}}^{(4)}$ the in-plane approximate $\langle 110\rangle$-directions are the easy

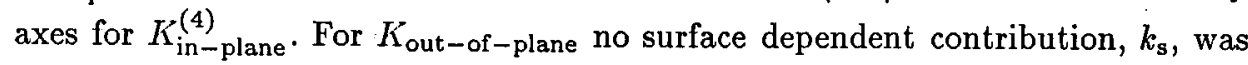
found, whereas the volume part was found to be $K_{\mathrm{s}}=(-5.0 \pm 0.6) \times 10^{6} \mathrm{erg} / \mathrm{cm}^{3}$. The negative sign indicates that the surface normal is a magnetic hard axis.

We now show that both $K_{\mathrm{p}}^{(2)}$ and $K_{\mathrm{s}}^{(2)}$ are caused by magneto-elastic interaction due to the elastic strain field originating from the lattice mismatch at the $\mathrm{Co} / \mathrm{Cu}$ interface. In a continuum approach we assume a smooth film, i.e., we neglect the stepped surface structure. Our approach is valid since the step distance of 6.5 atomic distances is much smaller than the static coherence length over which the magnetic moments might vary in direction. In the (1113) orientation the magneto-elastic tensor, $b_{i j k l}$, appearing in Eq. (4) must be rotated from the crystallographic reference frame into the film coordinate system with the cartesian

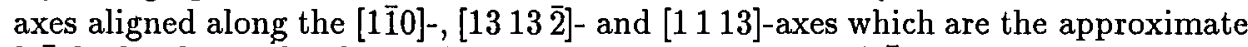
[110]-, [110]- and [001]-axes (i.e. here by $6.2^{\circ}$ about the [110]-axis). In the film coordinate system only the diagonal strain components $\varepsilon_{i i}$ are nonzero. The tensor components of the magneto-elastic tensor are obtained [6] from the magnetostriction constants $\lambda_{100}$ and $\lambda_{111}$ of fcc Co extrapolated from Co-rich CoPd alloys [24], as well as from the elastic constants of bulk fcc Co [28].

Figure 3 shows the obtained results. The values of the magneto-elastic aniso-

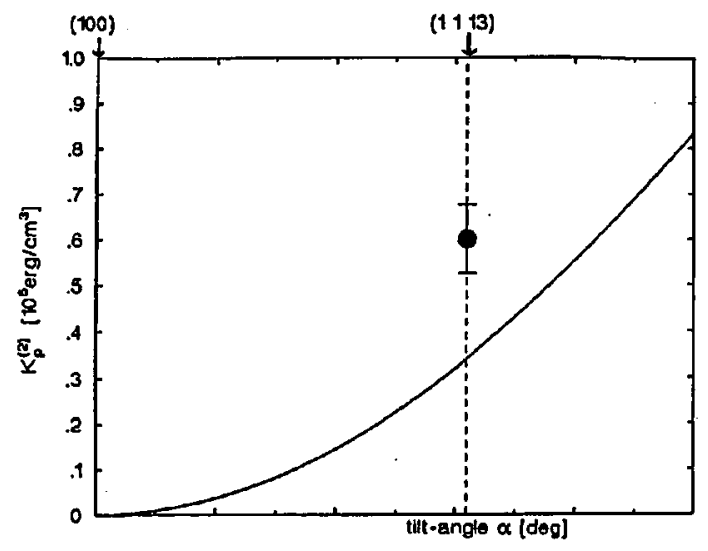

Fig. 3. Calculated in-plane $\left(K_{\mathrm{p}}^{(2)}\right)$ magneto-elastic anisotropy contributions as a function of the tilt angle $\alpha$, by which the surface is rotated about the in-plane [110]-axis out of the (001)-orientation. The (1 11 13)-orientation is marked by a dashed line. The experimental value for the (llllllion is shown as well. 
tropy constant $K_{\mathrm{p}}^{(2)}$ are plotted as a function of the tilt angle $\alpha$, by which the surface is rotated about the [110]-axis. For $\alpha=0$, i.e. for (100)-orientation, the in-plane constant, $K_{\mathrm{p}}^{(2)}$, is zero for symmetry reasons. With increasing tilt $K_{\mathrm{p}}^{(2)}$ increases. The experimental value of $K_{\mathrm{p}}^{(2)}$ for the (1113)-orientation $\left(\alpha=6.2^{\circ}\right)$ is shown as well. It agrees with the calculated value within a factor of two, which is a rather good agreement taking into account the uncertainty in the estimates of the magnetostriction constants as well as the approximation of using bulk elastic constants for the film. We therefore conclude that this anisotropy contribution is indeed caused by magneto-elastic interaction.

A careful investigation of Eq. (4) shows that the value and sign of $K_{\mathrm{p}}^{(2)}$ depend very sensitively on a possible strain relaxation. A relaxation of the lattice parameter in the in-plane direction perpendicular to the steps, as recently proposed [26], by $3 \%$ with no relaxation perpendicular to the steps would cancel $K_{\mathrm{p}}^{(2)}$, and a further relaxation would reverse the sign. From the low-energy electron diffraction (LEED) data we estimate that this type of relaxation is not present in our films although a relaxation of $3 \%$ is at the limit of resolution. We would also like to emphasize that in addition to the magneto-elastic contribution to the volume in-plane anisotropies, surface contributions might be caused by the large density of aligned steps on the surface [29], which might account for the experimentally observed value of the in-plane uniaxial surface anisotropy contribution, $k_{\mathrm{p}}^{(2)}$. However, a quantitative analysis of this contribution is presently out of reach.

\section{9. $\mathrm{Co} / \mathrm{Cu}(110)$ : evolution of magnetocrystalline bulk anisotropies}

In the film orientations previously discussed no hard evidence for the presence of magnetocrystalline anisotropies, as present in the bulk materials, was found. Magnetocrystalline contributions might be partly present in the "background" anisotropy contributions which could not further be identified in the film orientations discussed above.

In order to clarify this point (110)-oriented Co films prepared on $\mathrm{Cu}(110)$ were studied. The (110)-surface contains the [001]-, [110]- and [1111]-directions. Any in-plane surface anisotropy contribution would have either the [001]- or the [110]-axis as the symmetry axis for symmetry reasons. On the other hand, in fcc Co the magnetocrystalline bulk anisotropy favors the $\langle 111\rangle$-axes as the easy axes of magnetization. A study of the spin wave frequencies as a measure of the free energy of the system as a function of the in-plane direction of the applied field, $\phi_{H}$, thus can provide information about the contributing anisotropies.

Figure 4 shows the measured spin wave frequencies as a function of $\phi_{H}$ in an applied field of $3 \mathrm{kOe}$ for film thicknesses of $20 \AA$ and $100 \AA$. For $d_{\mathrm{Co}}=20 \AA \mathrm{max}-$ ima in the spin wave frequencies, which indicate the easy axis of magnetization, are found at $\phi_{H}$ equal to $0^{\circ}, 180^{\circ}$ and $360^{\circ}$ clearly exhibiting a twofold in-plane symmetry. For $d_{\mathrm{Co}}=100 \AA$ maxima are found at the in-plane $\langle 111\rangle$-directions as a clear fingerprint for the presence of magnetocrystalline anisotropies. A detailed analysis, performed by fitting simultaneously $K_{\text {in-plane }}^{(2)}, K_{\text {out-of-plane }}^{(2)}$ and the cubic magnetocrystalline bulk anisotropy constant, $K_{1}$, yields a value of $K_{1}=$ $-8.8 \times 10^{5} \mathrm{erg} / \mathrm{cm}^{3}$ in consistency with the high-temperature bulk value of fcc Co. 


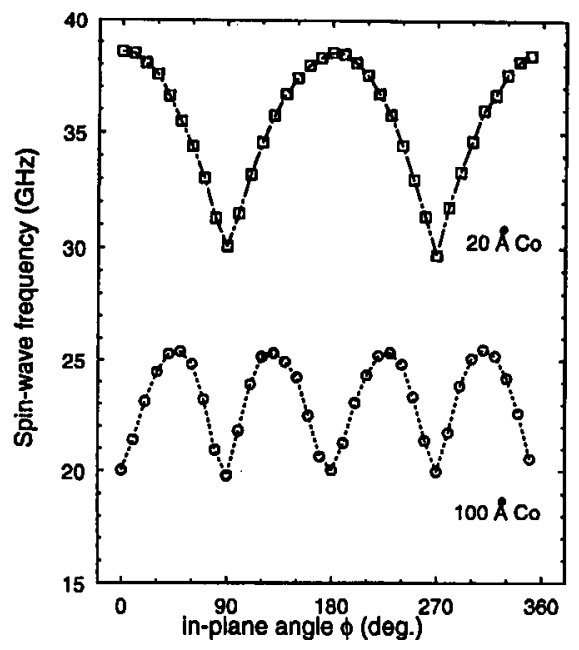

Fig. 4. Dependence of the measured spin wave frequencies on the in-plane direction of the external field of $3 \mathrm{kOe}$. $(\mathrm{Cu} / \mathrm{Co} / \mathrm{Cu}(110))$ maxima in the spin wave frequencies indicate an easy direction of the magnetization.

For $d_{\text {co }}>50 \AA$ the value of $K_{1}$ was found to be independent of film thickness. For smaller film thicknesses $K_{1}$ converges rapidly to zero. For film thicknesses below $40 \AA$ the value of $K_{1}$ is reduced to about $10 \%$ of its thick-film value.

\section{Conclusions}

In conclusion we have determined all contributing anisotropies in (111)-, (100)-, (1113)- and (110)-oriented Co films. In Co(1113), due to the induced twofold symmetry, a uniaxial in-plane anisotropy contribution is found in addition to the anisotropies found in (001)-oriented films. The origin of this anisotropy was found to be magneto-elastic. In addition, the out-of-plane volume anisotropy contribution was found to be consistent with a magneto-elastic origin, although other mechanisms like a magnetocrystalline contribution might contribute as well.

The obtained results offer access to the origin of the stabilization of ferromagnetic order in (001)- and (1113)-oriented films. As first discovered for (001)-oriented Co layers the stabilization mechanism is closely connected to the in-plane anisotropy. We find the same result both for the in-plane anisotropy contributions of twofold and fourfold symmetry. Since the former could be identified as being of magneto-elastic origin the elastic strain fields are likely to provide the driving force for the stabilization of ferromagnetic order in ultrathin Co films. Unfortunately, the origin of the in-plane anisotropy contribution of fourfold symmetry is inaccessible since higher-order magnetostriction constants of fcc Co would be required for a calculation of this contribution.

Magnetocrystalline bulk anisotropy contributions were identified in (110)-oriented Co films of film thicknesses larger than $50 \AA$. The suppression of this anisotropy contribution for smaller film thicknesses is presently not understood. It 
might be indicative for the presence of coherent resonance phenomena of electronic states in ultrathin films.

\section{Acknowledgments}

We would like to thank H.P. Oepen for discussions and supply of the Co(1 11 13)-substrates. This work was supported by the Deutsche Forschungsgemeinschaft through SFB 341.

\section{References}

[1] L. Néel, J. Phys. Radium 15, 225 (1954).

[2] C. Chappert, P. Bruno, J. Appl. Phys. 64, 5736 (1988).

[3] P. Bruno, J. Phys. E 18, 1291 (1988).

[4] G.T. Rado, J. Appl. Phys. 61, 4264 (1987).

[5] U. Gradmann, J. Korecki, G. Waller, Appl. Phys. A 39, 101 (1986).

[6] B. Hillebrands, J.R. Dutcher, Phys. Rev. B 47, 6126 (1993).

[7] See, e.g., B. Hillebrands, G. Güntherodt, in: Ultrathin Magnetic Structures, Vol. II, Eds. J.A.C. Bland, B. Heinrich, Springer Verlag, Heidelberg 1993, in print.

[8] B. Hillebrands, Phys. Rev. B 41, 530 (1990).

[9] R.L. Stamps, B. Hillebrands, Phys. Rev. B 44, 12417 (1991).

[10] P. Krams, F. Lauks, R.L. Stamps, B. Hillebrands, G. Güntherodt, Phys. Rev. Lett. 69, 3674 (1992).

[11] P. Krams, B. Hillebrands, G. Güntherodt, H.P. Oepen, to be published.

[12] M.T. Johnson, J.J. de Vries, N.W.E. McGee, J. aan de Stegge, F.J.A. den Broeder, Phys. Rev. Lett. 09, 3575 (1992).

[13] R. Mock, B. Hillebrands, J.R. Sandercock, J. Phys. E 20, 657 (1987).

[14] S.T. Purcell, M.T. Johnson, N.W.E. McGee, W.B. Zeper, W. Hoving, J. Magn. Magn. Mater, 113, 257 (1992).

[15] J. Kohlhepp, H.J. Elmers, U. Gradmann, J. Magn. Magn. Mater. 121, 487 (1993).

[16] N.D. Mermin, H. Wagner, Phys. Rev. Lett. 17, 1133 (1966).

[17] Y. Yafet, J. Kwo, E.M. Gyorgy, Phys. Rev. B 33, 6519 (1986).

[18] D. Pescia, V.L. Pokrovsky, Phys. Rev. Lett. 65, 2599 (1990).

[19] P. Bruno, Phys. Rev. B 43, 6015 (1991).

[20] J.A.C. Bland, G.A. Gehring, B. Kaplan, to be published.

[21] M.G. Pini, A.Rettori, D. Pescia, N. Majlis, S. Selzer, Phys. Rev. B 45, 5037 (1992).

[22] P. Krams, F. Lauks, R.L. Stamps, B. Hillebrands, G. Güntherodt, J. Magn. Magn. Mater. 121, 483 (1993).

[23] For $T>T_{\mathrm{c}}$ ultrathin Co films beha ve like ideal Heisenberg magnets, see: D. Kerkmann, D. Pescia, R. Allenspach, Phys. Rev. Lett. 68, 686 (1992). Thus in our case for $d<d_{\mathrm{c}}$ a rather large magnetic moment might be induced by an applied magnetic field.

[24] H. Fujiwara, H. Kadomatsu, T. Tokunaga, J. Magn. Magn. Mater. 31-34, 809 (1983). 
[25] A. Berger, U. Linke, H.P. Oepen, Phys. Rev. Lett. 68, 839 (1992).

[26] II.P. Oepen, C. Schneider, D.S. Chuang, C.A. Ballentine, R.C. O'Handley, J. Appl. Phys. 73, 6186 (1993).

[27] H.P. Oepen, A. Berger, C.M. Schneider, T. Reul, J. Kirschner, J. Magn. Magn. Mater. 121, 490 (1992).

[28] Elastic, Piezoelectric, Pyroelectric, Piezooptic, Electrooptic Constants, and Nonlinear Dielectric Susceptibilities of Crystals, Eds. K.-H. Hellwege, A.M. Hellwege, Landolt-Börnstein, New Series, Group 3, Vol. 18, Springer-Verlag, Berlin 1984.

[29] M. Albrecht, T. Furubayashi, U. Gradmann, W.A. Harrison, J. Magn. Magn. Mater. 104-107, 1699 (1992). 